\title{
Critical Analysis of Dispersion Compensated Optical Communication System
}

\author{
R K Sethi \\ Research Scholar \\ Deptt. of Elex.\& Comm. Engg. \\ MANIT, Bhopal. India.
}

\author{
Aditya Goel \\ Professor \\ Deptt. of Elx.\& Comm. Engg. \\ MANIT, Bhopal. India
}

\begin{abstract}
Next generation communication systems need to dispense higher data rate in the efficient manner with capability to customize according to the dynamic data transfer framework in an economically dominant approach. The optical fiber communication networks fulfill these requirements along with small attenuation loss and better quality of services. In the last a few years the rapid progress of the data rate transmission over the existing optical transmission networks accentuated the need of more worthwhile usage of the transmission capacity of the prevailing networks. The forthcoming optical communication systems needs to support $10 \mathrm{Gbps}$ and above over the single wavelength in the optical fiber channel. The Inter-Symbol Interference (ISI) due to dispersion limits the repeater less maximum transmission data rate and maximum transmission distance for the optical fiber channel [1]. Various endeavors have been taken for the advancement of the schemes and dispersion compensation devices to mitigate the effect of the dispersion causing inter-symbol interference. The primary instrument to achieve this objective is to use an equalizer. Orthogonal Frequency Division Multiplexing $(\mathrm{OFDM})$ is a very favorable scheme for the high data rate data transmission because of its ability to overcome the effect of the dispersion, tolerance to the dispersive channel, high spectrum efficiency and better flexibility of operation. For mitigating dispersion impact OFDM transmits a large number of modulated sub-carriers at the same time operating at low data rate. These cause the symbol period relatively longer than the channel impulse response, hence mitigating the impact of inter-symbol interference. OFDM is a multicarrier modulation scheme, extensively investigated and deployed in wireless as well as wire line communication. It is getting increased interest in the fiber optic research community for its robustness against dispersion causing inter-symbol interference. It utilizes the spectrum efficiently and dynamically controls the dispersion. This suggest that OFDM can be the best method for the mitigating the dispersion for the long distance high data rate optical fiber communication systems. Therefore, the integration of OFDM with the optical fiber can be the excellent scheme for providing long distance high data rate efficiently. A comparative performance analysis of the dispersion compensated optical system carried out for examining the impact of the OFDM to mitigate the dispersion at $10 \mathrm{Gbps}$ and above. The systems with and without using OFDM has been configured and its performance has been investigated,various results obtained exhibited that transmission performance is entirely dependent upon a proper selection of data rate, transmission length, and modulation schemes etc. For evaluating the systems performance, constellation and bit error rate are evaluated for system with and without using OFDM and subsequently compared.The various results of the investigation depicts that for high data capacity transmission for the long distance the systems with
\end{abstract}

using OFDM shows better results, and having better spectrum utilization than the systems without using the OFDM.The use of a large number of sub-carriers has been observed to be more effective in overcoming the fibre dispersion.

\section{General Terms}

Optical Communication Systems, Optical Fiber, Dispersion Compensation Technique, Digital Signal Processing.

\section{Keywords}

OFDM, Dispersion, SSMF, PMD, GVD, IFFT, FFT.

\section{INTRODUCTION}

The principal objective of the communication systems is to communicate to the longest possible distance and maximum possible data rates with lowest bit error rates. These requirements, along with the good quality of the transmission can be achieved by utilizing the optical fiber networks, operating at higher data transmission rate and low attenuation loss [9].The high transmission data rate operation causes inter-symbol interference due to dispersion; it must be eliminated in economically viable manner for getting error free transmission.Dispersion thus limits the maximum data transmissionrate through fiber optic channel. Due to intersymbol interference it restricts bit interval period and the highest data transmission rate on an optic al fiber channel.This limitation also restricts the maximum span of the fiber for introducing regenerator in the optical fiber link [1].From the dispersion characteristics of the standard single optical fiber it may be noted dispersion at the wavelength of $1550 \mathrm{~nm}$ has comparatively higher values than at $1310 \mathrm{~nm}$ on standard single mode fiber.Hence the operation of the standard single mode fiber installed earlier, at $1550 \mathrm{~nm}$ offer comparatively higher dispersion than at $1310 \mathrm{~nm}$. The short distance communication systems operating at $2.5 \mathrm{Gbps}$ and less the dispersion are not restricting parameters. As the demand for more bandwidth per channel increases, causing migration to the $10 \mathrm{Gbps}$ data rate transmission. But for long distance communication systems' operating at $10 \mathrm{Gbps}$ and more the dispersion causing inter-symbol interference is seriously affecting the transmission performance which must be suitably eliminated. Hence it restricts its use for the high data rate of $10 \mathrm{Gbps}$ and above for the long distance optical fiber communication networks. In order to keep pace with the increasing demand of communication bandwidth, and provide the service cost efficiently, engineers have turned to the development of higher speed systems for longer distance. While the power attenuation task is overcame by the invention of optical amplifiers, dispersion causing pulse spreading become the dominant limit of transmission speed and distance of optical communications. The main obstacle concerned for exiting optical fiber networks are dispersion causing intersymbol interference, they need to be replaced for high data rate transmission applications. The complete replacement of 
the previously installed optical fiber transmission network is not possible mainly because of the economic reason. Therefore, the dispersion should be suitably compensated for using the existing network for high data rate application.

\subsection{Optical Communication System}

The principal objective of any transmission is to provide excellent signal quality for the maximum possible distance. Fiber optic transmission media is the excellent for transmitting high speed information with very low losses and cost effectiveness. Principally, single mode fiber can transmit data at the rate of about 50 Tbps; hence with optical fibers we can get network link capacities of the order of thousands of Gbps [14]. The first concept of long hauloptical communication using a glass fiber utilizing optical pulses was initiated by Hockman and Kao in 1966 [12]. Initially, it was materialized when Coming fabricated low loss glass optical fibers [13] and at the same time Bell labs developed the semiconductor diode lasers [14]. The elementary optical system consists of transmitter, to produce modulated optical signal, optical fiber as a transmission channel receiver, to convert optical signal in to the transmitted information data and the power amplifier to amplify the weak optical signal in the channel. For more sophisticated optical systems, some more components are required.The level of the complexity of the same can vary from simple local area networks to highly complex long haul telephone or cable TV network[16].Dispersion causing the inter-symbol interference limits the maximum data rate transmission capacity of the fiber optic channel. It was not limiting factor for the systems operating at $2.5 \mathrm{Gbps}$ and less data rate for short distance transmission. With the increase in the demand for higher data rate and subsequently the migration to the $10 \mathrm{Gbps}$ and above data rate the dispersion becomes the prime limiting factor. It fixes the boundary lines for the bit interval and the maximum data rate on an optic al fiber channel. It needs to be suitably compensated for error free transmission. For the optical fiber channel the main causes for the dispersion are group velocity dispersion i.e., different frequencies components propagates at varying velocity and polarization mode dispersion i.e., the two polarize optical signal components reaches the receiver with varying delays. The effect of dispersion increases proportionately with data transmission rate, since dispersion permissiveness decays proportionately with the square of the data bit rates [15]. With the rise in the need of different voice, video, images, and data services, the urge for communication networks to get the same also progressed. From the various available technologies the combination of the optical signal and optical fiber gives a huge data transmission capacity compared to any other systems. Consequently, the end connection for the subscribers for accessing various applications can be wireless or wired; however, the main distribution of the data between links is dependent on the optical fiber. Distinctly, optical fiber communication will be the leading technology for these requirements.

\subsection{Dispersion Compensation Techniques}

In order to keep pace with the increasing demand of communication bandwidth, and provide the service cost efficiently, engineers have turned to the development of higher speed systems for longer distance. While the power attenuation task is overcame by the invention of optical amplifiers, dispersion causing pulse spreading become the dominant limit of transmission speed and distance of optical communications. As the transmission rate goes from OC-48 (2.5 Gbps) to OC-192 (10 Gbps) or even OC-768 (40 Gbps), the dispersion compensation requirement will only become more critical for system, to use the optical cables installed during the economic boom, since they do not have any operational margin to afford new requirement of the higher data rate transmission. The main obstacle concerned for the previously installed optical fiber are that they are obsolete (out dated), hence they offer comparatively higher values for different restricting parameters like, chromatic and polarization mode dispersion. One of the best solutions to remove the restriction in high speed data transmission due to dispersion is to re-install previously installed optical network with latest optical fibre. Due to the economic reasons, this solution is not practicable, and therefore we must deal with the installed old fibre and cope with the relative problems. Hence, the main limitation in releasing the full bandwidth capacity of an optical fiber is pulse distortion due to dispersion. High data rate leads to inter-symbol interference due to dispersion. It fixes the boundary lines for the bit interval and the maximum data rate on an optical fiber channel. The standard single mode fibre has minimum dispersion but comparatively higher attenuation at $1310 \mathrm{~nm}$ wavelength; whereas it has lowest attenuation at $1550 \mathrm{~nm}$ wavelength but the dispersion is higher than the 1310 nm.Hence it is required to compensate the high value of the dispersion at $1550 \mathrm{~nm}$ wavelength by using the dispersion compensating optical fiber with very high value of the negative dispersion, so as to compensate the same.The problem of dispersion can be mitigated by inserting an dispersion compensating element that imposes dispersion on the optical signal that is opposite to that imposed by optical fiber channel [2, 21, 22]. By specially designing optical fibers one can efficiently producesufficiently high value of negative dispersion in the system, subsequently nullify the overall dispersion of the system for long range of the frequency spectrum. It can actually reverse the effects of dispersion suffered by $1550 \mathrm{~nm}$ signals that traverse standard singlemode fiber. It is used as a sort of inline pre- or postequalization in the form of a fiber spool of a particular length placed at one end of a link. But it has the disadvantage of high cost, physical size, signal delay and lack of adaptability. The attenuation of the dispersion compensating fibre requires additional optical amplifiers, which introduce additional optical noise $[23,36]$. It is a simple way to compensate for dispersion to cancel the accumulated dispersion after a certain distance by mean of the dispersion compensating fibre. In this scheme a suitable length of the dispersion compensation fiber having strong negative dispersion [8], placed at regular intervals along the link usually at the optical amplifier points, typically at 80-130 km. Mcnicol and Killey [21, 22] suggested that interest in electronic dispersion compensationis rising due to its adaptability to any optical fiber communication system, including dynamically-switched optical networks, so reduces engineering and inventory costs. Also it reduces the need of the repeaters along the transmission path. By employing electronic dispersion compensation the data transmission capacity of the existing network without changing architecture of the network, but raises the burden of digital signal processing. Hence, it implementation will be more cost effective [27]. Similarly, electronic pre-distortion [27-28] is a modern developed version of the electronic dispersion compensation, however this techniques needs a feedback from the receiving end [4].Apart from dispersion compensating fibre [18-21], several other different dispersion compensation methods like, fibreBragg gratings [22-24], all-pass optical filters [16] and optical phase conjugation [19-21] reverse dispersion fiber and negative dispersion fiber [37-38]. All 
these techniques reconditioned the optical signal for retrieving the information with normal receiver. But, the implementations of the various schemes are not economically satisfactory for the previously installed existing systems. The use of the regenerator along with the transmission path is not economically viable for the long distance optical networks.

\subsection{Orthogonal Frequency Division Multiplexing}

OFDM is a special form of multicarrier modulation scheme in which various orthogonal sub-carriers having partially overlapped spectrum.OFDM is a very favorable scheme for the high data rate transmission, because of its ability to mitigate the effect of the dispersion, tolerance to the dispersive channel, high spectrum efficiency and better flexibility of operation. For mitigating the impact of dispersion, it transmits a large number of parallel modulated sub-carriers simultaneously at low data rate. These results in the symbol period of the channel comparatively longer than the impulse response of the channel thereby mitigate the intersymbol interference. It permits proficiently and reliably data transfer over a dispersive radio channel, even in multipath atmosphere. OFDM is a multicarrier modulation technique that has been extensively investigated and deployed in wireless and wire line communication. It is receiving increased interest in the fiber optic research community for its robustness against inter-symbol interference. The OFDM utilizes the spectrum efficiently and control the dispersion dynamically by simultaneously using a large number of orthogonal sub-carriers, it suggesting that OFDM can be the one of the best techniques for the dispersion compensation for the long distance high data capacity optical fiber communication systems. Hence it will allow the use of OFDM in previously installed optical fiber communication systems / networks for longer distance. Therefore, integration of OFDM with the optical fiber can be the excellent scheme for the high data rate long distance transmission in a very efficient manner. The basic idea for employing OFDM for high data rate communication was originated by Chang in 1966 at Bell Labs [31]. Subsequently in 1969 and 1970 the major advancement of OFDM was introduced by Weinstein et al. [32]. They articulated the application of inverse Fourier transform and Fourier transform, as a viable scheme for the implementation of the OFDM. Because of the different restriction it was not executed for the different utilities, even though got patent in 1070.In 1990 Cioffi et al. [33-35] at Stanford University implemented the same in the wire line protocol ADSL. Latter, Dixon et al. suggested its utility for mitigating dispersion in optical fiber [8]. The OFDM resilience to the dispersionpermits high data rate transmission;thereby it is recommended for the next generation networks in dispersive environments [14]. It have been accepted and implemented in different wireless wide band data transmission schemes like, mobile telephones, satellite links, digital audio / video transmission, [11], wireless local/metropolitan area networks standards IEEE 802.11a/g Wi Fi, IEEE 802.16 WiMAX, general switched telephone network, digital subscriber lines.

\section{DISPERSION COMPENSATION IN OPTICAL COMMUNICATION SYSTEM USING OFDM}

For fulfilling the urge of the different communication application of the modern era, the optical fiber communication system networks havehuge bandwidth capacity. There is deterioration in the receivedsignal in optical communication systems due to signal attenuation and signal dispersion, making it very cumbersome to retrieve thetransmitted data from the received signals, resulting in the loss in information. By using Erbium Doped Fiber Amplifier (EDFA) one can compensate the attenuation loss along the optical fiber link, but the dispersion causing inter-symbol interference needs special compensation techniques. Therefore for the high capacity long distance transmission through optical fiber channel the impact of the dispersion needs to be eliminated.The OFDM widely employed in wired and wireless systems, it overcomes the problem of the dispersion very efficiently. The OFDM allows the transmission of the high data rate signal efficiently by using multiplexing of alarge number of sub-carriers in the multipath environment, credibly because of its flexibility to the dispersion. In an OFDM transmission scheme, there is simultaneously transmission of large number of sub-carriers which are orthogonal, narrow band and overlapping. It divides the available transmission bandwidth into narrow band. The sub-carrier partitions of the OFDM technique are theoretically lowest and split the existing bandwidth in such a way that all sub-carriers are mathematically orthogonal. Because of the orthogonality there is not any inter-carrier interference among sub-carriers. Consequently the utilization of the spectrum is efficient. The phrase mathematically orthogonality generally means sub-carrier are placed in such a way that it comes at the null energy point. Various OFDM sub-carriers are modulated at comparatively small symbol rate, making the symbols duration much longer compare to the optical channel impulse response, this will eliminate the ISI. By prefixing extra guard band between adjacent OFDM symbols it can decrease the impact of ISI even further. Though this guard band is comparatively longer than the multipath delay, the impact of ISI can totally neutralize. There is a loss of the data capacity due to the low data rate over sub-carrier, in order to compensate the same;simultaneous very large sub-carriers are utilized, resulting in a very high data transmission rate. These forces that the inter-symbol interference due to dispersion is very less or almost null on the performance of the OFDM systems, therefore the equalizer are not required at the receiver. If the delays due to multipath propagation do not surpass the guard interval, the ISI will not occur and channel equalization is not needed. The resilience of OFDM to multipath delay in the dispersive radio frequency channel recommends that OFDM can be endurable to the impact of dispersion in optical fiber. Hence it will permit the use of earlier installed optical fiber communication networks for longer distance. OFDM has recently become of great interest to the optical communications research community. For the various application of the OFDM many research centers in the world have specialized teams working. The OFDM is highly sensitive to phase noise, also has a comparatively higher peak power to average power ratio [10], which causes higher susceptible to fiber nonlinearities; so to take the complete benefit and minimize negative impact of OFDM, a careful design of a system using various parameters of the OFDM is needed.By employing the forward error detecting and correction and time / frequency interleaving the system enhanced its performance to repair erroneous sub-carriers. Additionally, the same can be further improved by increasing number of sub-carriers \& the guard band interval and employing sophisticated modulation techniques. OFDM provides dynamic dispersion tolerance along with flexibility among the various parameters like, number of sub-carriers, modulation schemes and cyclic prefix as a function of the 
peculiarity of the communication channel. Therefore, for long distance communication the integration of OFDM with the optical fiber can be the excellent scheme for providing higher data rate in the efficient manner.

\section{MODELING AND SIMULATION}

For the next generation communication network the data rate projected is $10 \mathrm{Gbps}$ and above. We investigated the practicability of $10 \mathrm{Gbps}$ and beyond data rate transmission over optical fiber system.This investigation optical communication system iscentered on dispersion compensation \&spectral efficiency properties of the OFDM when no dispersion compensation is employed. It overcomes the problem of dispersion by simultaneously transmitting a large number of orthogonal sub-carriers, to substantiate the same we have conducted analysis on optical fiber system with \& without using OFDM and compared the performance. Various results depicts that the effective dispersion compensation can be obtained by employing OFDM techniques in the existing optical communication systems, and one can get overall data rate up to $40 \mathrm{Gbps}$. In this work optical communication systems simulated for examining the capacity of the OFDM to mitigate the impact dispersion, for the system which does not incorporate any external dispersion compensation sub-system.

A brief description of the major steps in simulation $[3,38]$ of the same systemsis given in the next section. The dispersion compensating capability of the optical fiber systems with \& without employing OFDM evaluated and comparedby parameter, like the signal constellation / BER at the receiver for varying fiber lengths, data transmission rates, etc. The simulated results are depicted as constellation diagrams / BER plots for the optical systems at varying fiber lengths from 0$200 \mathrm{Km}$ at centered wavelength of $1550 \mathrm{~nm}$, with and without employing OFDM. These parameters have taken as the performance index in the subsequent analysis. For the performance evaluation, the systems are simulated by using the software Optisystem and Matlab already utilized by different research institute and research scholars for the same $[30,39]$.

\subsection{Optical Communication System using OFDM}

The different sub-systemsas depicted in Figure3.2are OFDM modulator, optical transmitter, optical fiber channel, optical receiver, \& OFDM demodulator. The sub-carriers are modulated using 16-QAM modulation format. For the examination of the performance the systems has been configured for the fiber span ranging 0-200 Km, at the centre wavelength $1550 \mathrm{~nm}$, data rate 1-40 Gbps and sub-carriers varying from 256-512-1024. The number of sub-carriers, data rate, cyclic prefix \& the type of sub-carrier modulation are significant parameters of the OFDM system influencing the transmission capacity and the distance of optical communication systems. The fundamental parameters, like dispersion, noise etc., of the systems are incorporated in the modeling and simulation.

The Pseudo-Random Binary Sequence (PRBS) outputs are mapped for 16-QAM modulation. By the cyclic prefix one can additionally eliminate the dispersion effect causing intersymbol interference. The 16-QAM encoded PRBS data subcarriers are OFDM modulated using OFDM transmitter consisting of the IFFT. The IFFT transfer the signal from frequency to time domain. For the coherent optical modulation of the OFDM data signal,in-phase and Quadrature phase parts are utilized. For optical modulation is performed by applying the OFDM signal to an optical "I-Q" modulator biased at null point, by employing Mach-Zehnder (MZ) modulator along with the CW Laser[14, 16]. This is followed by the digital to analogue converter.

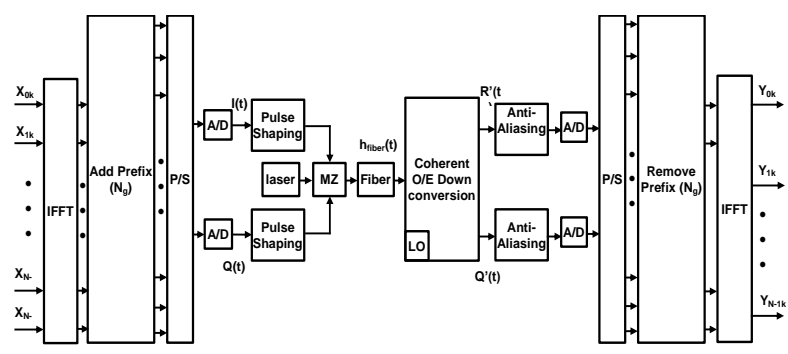

Figure 3.1: Optical Communication System using OFDM

Subsequently, OFDM modulated optical signal centered at $1550 \mathrm{~nm}$ wavelength are fed to the optical fiber channel.Itconsisting of a number of loops of the standard single mode optical fiber and erbium doped fiber amplifiers. Fiber spans having the signal attenuation loss of $0.2 \mathrm{~dB}$ per $\mathrm{Km}$, dispersion coefficient at centered wavelength $1550 \mathrm{~nm}$ is 17 ps per nm per Km and fiber nonlinearity coefficient of 2.09 per watt per $\mathrm{Km}$. Contradictory to the traditional scheme of optical dispersion compensationOFDMdoes not requires any separate module for dispersion compensation along with the optical channel. In optical fiber channel the signal gets corrupted due to the dispersion.At the receiving end for coherent detectionis performed to detectthe "I and Q" component of the OFDM signal. coherent detector composing of two pairs of balanced photo-detectors, an optical 90 degree hybrid, and a local oscillator laser, which helps optical to radio frequency OFDM direct down conversion. Here, optical receiver noise likes, thermal noise, shot noise, etc. are considered. Optical OFDM signal after conversion electrical form sent to analog to digital converter at the receiver followed by the OFDM demodulator. These down converted "I and Q" components are OFDM demodulated and decoded using 16-QAM de-mapped for extracting the transmitted data, for examining the system performance.Then the signal 16QAM demodulated and decoded to get the original transmitted information. For the performance evaluation by systems parametersthe received signals are used to compute various transmission parameters and depicted graphically as constellations diagrams / BER. Afterward, the same are compared for different fiber lengths, data rate, and number of sub-carriers etc. at receiving end.There is need not to pay attention to the cross phase modulation and four wave mixing as they are not produced in the single channel operation. The focus of the investigation is to examine the dispersion compensating capacity of the OFDM to mitigate the impact of dispersion in optical communication system.

\subsection{Optical Communication System without using OFDM}

The optical system employing 16-QAM without using OFDM centered at $1550 \mathrm{~nm}$ wavelength, demonstrated in Figure 3.2. It is modeled and simulated for the comparisons purpose only. The 16-QAM mapped PRBS data is modulated into optical signals by utilizing MZ modulator. Subsequently these signals are sent through the fiber channel, having EDFA to overcome the attenuation. The optical modulated output signals at the receiving end fed to optical receiver, for optical to electrical 
conversion. Afterward, low frequency pass Bessel filter used for noise reduction and smoothing of the electrical signal low pass Bessel filters are used. It is comprises of the 16-QAM modulator, optical signal modulator / transmitter, fiber channel, optical signal receiver / demodulator and 16-QAM demodulator. For the examination of the performance the systems has been configured for the fiber span ranging 0-200 $\mathrm{Km}$, at centered $1550 \mathrm{~nm}$ wavelength for data rate varying from 1 to $40 \mathrm{Gbps}$. The simulation setups used for comparison is having the same set of parameters like data rate, modulation formats, span of optical fiber etc. The dispersion compensating capacity of the systems without OFDM assessed for comparisons with systems using OFDM, by evaluating the transmission parameter, constellation and bit error rate at the receiver for varying fiber lengths, data rates, etc. The results for both the systems presented graphically as constellationand bit error rate plot for system for fiber span varying $0-200 \mathrm{Km}$.

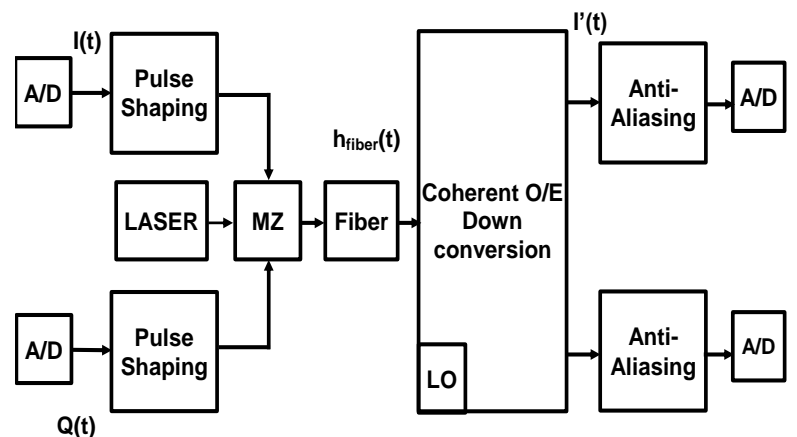

$Q^{\prime}(t)$

Figure 3.2: Optical Communication System without using OFDM

\section{RESULTS AND DISCUSSIONS}

Here, performance analysis of system carried out for examining the effect of the OFDM to eliminate the dispersion. The system with \& without using OFDM has been configured and their performance compared, various results exhibits that transmission performance is entirely dependent upon a proper selection of data rate, transmission distance, and modulation schemes etc. The systems performance have been evaluated by computing the bit error rate $\&$ constellation and compared for data rate ranging 1.0-40 Gbps, centered at $1550 \mathrm{~nm}$ wavelength for fiber span ranging $0-200 \mathrm{Km}$ and number of sub-carriers etc. The various simulated results are shown graphically. Figure 4.1 depicts the transmitted 16-QAM constellation.

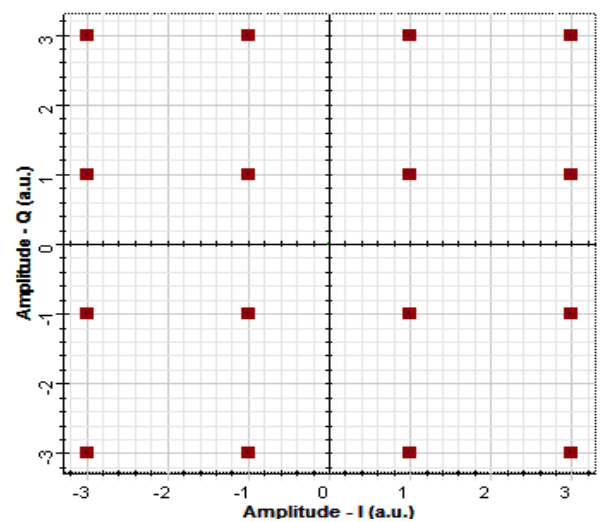

Figure 4.1: Input SignalConstellationsof 16-QAM

\subsection{Performance Evaluation of Optical Communication Systems by employing 16- QAM with using OFDM}

\subsubsection{OAM OFDM 256 Sub-carriers 10Gbps}

The different results are computed and shown graphically in the Figures 4.2-4.3 as constellation diagrams / BER graphs for the system at different fiber lengths from 0-200 Km at the centre wavelength of $1550 \mathrm{~nm}$, with / without using OFDM. From the constellation diagrams for the optical systems with 256 sub-carriers transmission with fiber span ranging 0-200 $\mathrm{Km}$ for data rate $10 \mathrm{Gbps}$, it is found that for initial $150 \mathrm{Km}$ quality of the received signal is reasonably good and afterward it gets distorted due to the dispersion. The various symbols in constellation gets closer \& closer, causing the higher bit error rate, which in turn it limits the transmission performance of the system. Simultaneously, the BER also deteriorates as shown if the Figure4.3. Subsequently this distortion due to the dispersion causes higher bit error rate. Therefore the data transmission capacity of the systems deteriorated. Hence, for keeping the distance data rate product constant one should reduce either data rate or fiber span. Therefore, theanalysis of systemsperformance by bit error rate \&constellationsuggests that the overallsystem performance with 256sub-carriersoperating at $10 \mathrm{Gbps}$ isfairly good and having tolerable bit error rate for the initial $150 \mathrm{Km}$ and afterward itdegrades.

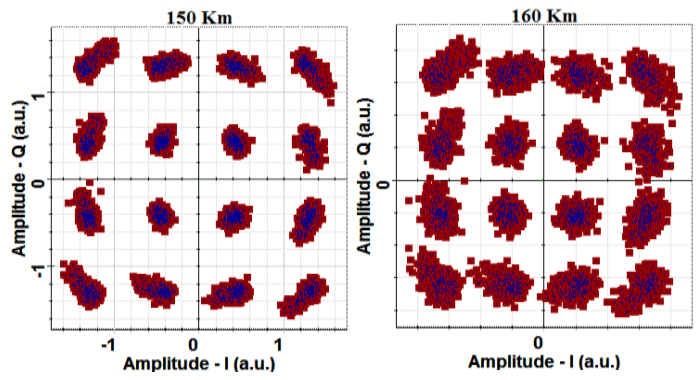

Figure4.2: Constellation for the Received Data Signal for OFDM 16-QAM 256 Sub-carriers at 10 Gbps.

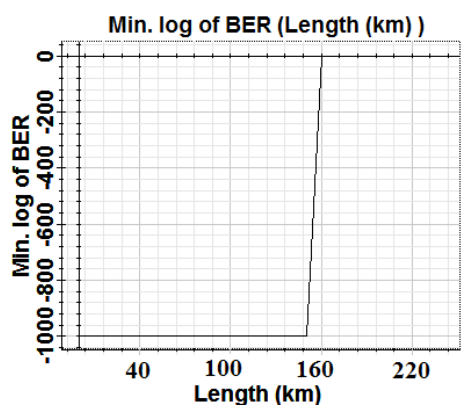

Figure4.3: Evaluation of BER V/s Length Obtained using OFDM 16-QAM 256 Sub-carriers at 10 Gbps.

\subsubsection{QAM OFDM 512Sub-carriers 10GBPS}

The various results are computed and presented graphically in the Figures 4.4-4.5 as constellation diagrams / BER graphs for the optical system at different fiber lengths from 0-200 $\mathrm{Km}$ at the centre wavelength of $1550 \mathrm{~nm}$, with / without using OFDM. From the constellation diagrams for the optical systems with 512 sub-carriers transmission with fiber span ranging $0-200 \mathrm{Km}$ for data rate $10 \mathrm{Gbps}$, it is found that for initial $160 \mathrm{Km}$ quality of the received signal is reasonably good and afterward it gets distorted due to the dispersion. The 
various symbols in constellation gets closer \& closer, causing the higher bit error rate, which in turn it limits the transmission performance of the system. Simultaneously, the BER also deteriorates as shown if the Figure 4.5. Subsequently this distortion due to the dispersion causes higher bit error rate. Therefore the data transmission capacity of the systems deteriorated. Hence, for keeping the distance data rate product constant one should reduce either data rate or fiber span. Therefore, the analysis of systems performance by bit error rate \& constellation suggests that the overall system performance with 512 sub-carriers operating at 10 Gbps is fairly good and having tolerable bit error rate for the initial $160 \mathrm{Km}$ and afterward it degrades.
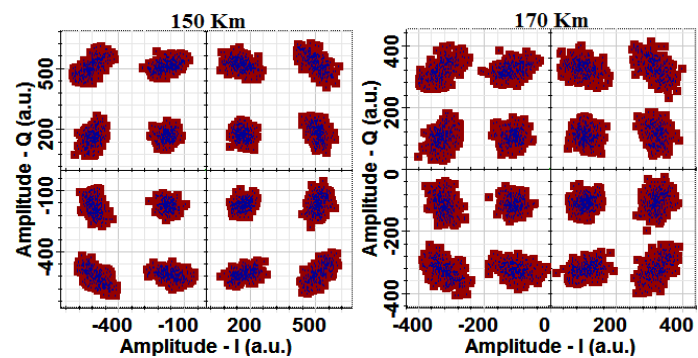

Figure4.4:Constellation for the Received Data Signal for OFDM 16-QAM 512 Sub-carriers at $10 \mathrm{Gbps}$.

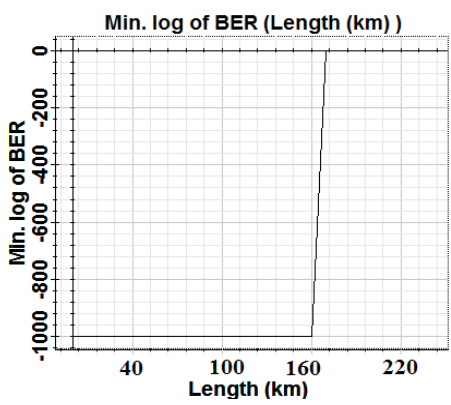

Figure 4.5: Evaluation of BER V/s Length Obtained using OFDM 16-QAM 512 Sub-carriers at $10 \mathrm{Gbps}$.

\subsubsection{QAM OFDM 1024 Sub-carriers 10Gbps}

The various results are computed and presented graphically in the Figures 4.6-4.7 as constellation diagrams / BER graphs for the optical system at different fiber lengths from 0-200 $\mathrm{Km}$ at the centre wavelength of $1550 \mathrm{~nm}$, with / without using OFDM. From the constellation diagrams for the optical systems with 1024 sub-carriers transmission with fiber span ranging $0-200 \mathrm{Km}$ for data rate $10 \mathrm{Gbps}$, it is found that for initial $160 \mathrm{Km}$ quality of the received signal is reasonably good and afterward it gets distorted due to the dispersion. The symbols in constellation gets closer \& closer, results in the higher bit error rate, which in turn it limits the transmission performance of the system. Simultaneously, the BER also deteriorates as shown if the Figure 4.7. Subsequently this distortion due to the dispersion causes higher bit error rate. Therefore the data transmission capacity of the systems deteriorated. Hence, for keeping the distance data rate product constant one should reduce either data rate or fiber span. Hence from the system performance evaluation by BER and Constellation, it can observe that the system performance at $10 \mathrm{Gbps}$ with 1024 sub-carriers is well within the acceptable level with permissible bit error rate for initial $160 \mathrm{Km}$ for system with using OFDM and beyond $170 \mathrm{Km}$ it is degraded.

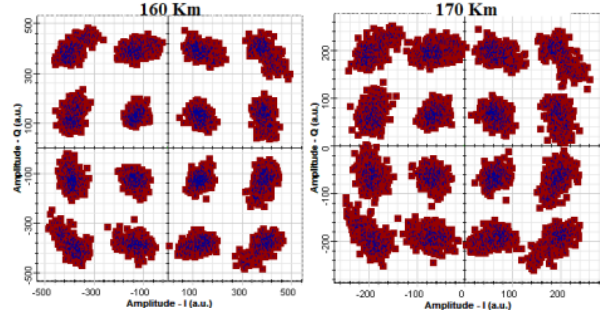

Figure4.6:Constellation for the Received Data Signal for OFDM 16-QAM 1024 Sub-carriers at 10 Gbps.

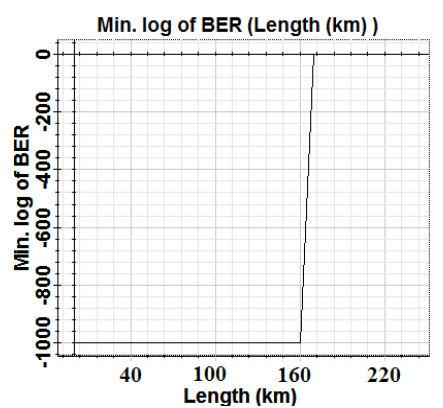

Figure4.7: Evaluation of BER V/s Length Obtained using OFDM 16-QAM 1024 Sub-carriers at 10 Gbps.

\subsubsection{QAM OFDM 256 Sub-carriers 20GBPS}

The different parameters are computed and presented graphically in the Figures 4.8-4.9 as constellation diagrams and BER graphs for the system at $20 \mathrm{Gbps}$ for fiber span ranging 0-200 $\mathrm{Km}$ centeredat $1550 \mathrm{~nm}$ wavelength, without using OFDM. From the constellation diagrams at $20 \mathrm{Gbps}$ with 256 sub-carriers for fiber span varying 0-200 Km, it is observe that for initial $160 \mathrm{Km}$ the signal quality is reasonably good and afterwards the received signal gets distorted due to the dispersion. The symbols in constellation diagram become closer \& closer, results in the higher bit error rate, which in turn it limits the transmission performance of the system. Simultaneously, the BER also deteriorates as shown if the Figure 4.8. Subsequently this distortion due to the dispersion causes higher bit error rate. Therefore the data transmission capacity of the systems deteriorated. Hence, for keeping the distance data rate product constant one should reduce either data rate or fiber span. Hence from the system performance evaluation by BER and Constellation, it seems that the system performance at $20 \mathrm{Gbps}$ with using 1024 sub-carriers is reasonably good with permissible bit error rate even for 70 $\mathrm{Km}$ of the fiber span for the data rate of $20 \mathrm{Gbps}$ system with using OFDM, beyond $80 \mathrm{Km}$ the performance is degraded.
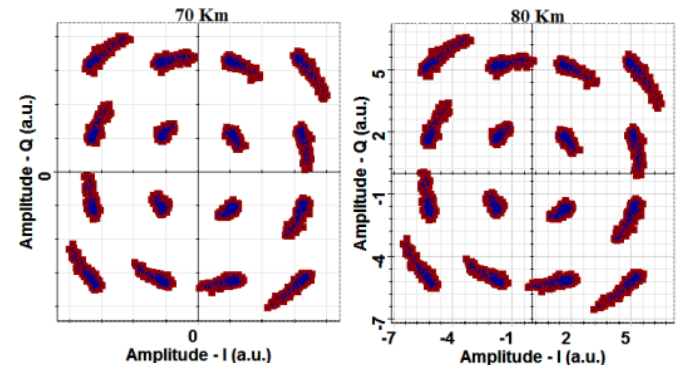

Figure4.8: Constellation for the Received Data Signal for OFDM 16-QAM 256 Sub-carriers at 20 Gbps. 


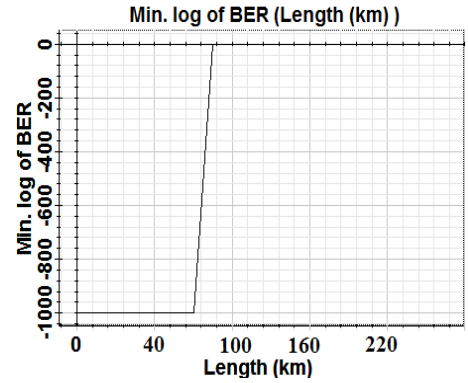

Figure4.9: Evaluation of BER V/s Length Obtained Through OFDM 16-QAM 256 Sub-carriers at 20 Gbps.

\subsubsection{6-QAM OFDM 512 Sub-carriers 20GBPS}

The various results are computed and presented graphically in the Figures 4.10-4.11 as constellation diagrams / BER graphs for the system at different fiber lengths from $0-200 \mathrm{Km}$ at the centre wavelength of $1550 \mathrm{~nm}$, without using OFDM. From the constellation diagrams for $20 \mathrm{Gbps}$ data rate with $512 \mathrm{sub}-$ carriers transmission with selected fiber length of 0-200 Km, it analyzed that up to $80 \mathrm{Km}$ the signal quality is reasonably good and as we further increase the length of the fiber the received signal gets distorted due to the dispersion. The various symbols in the constellation diagram becomes closer \& closer, results in the higher bit error rate, which in turn it limits the transmission performance of the system. Simultaneously, the BER also deteriorates as shown if the Figure 4.11. Subsequently this distortion due to the dispersion causes higher bit error rate. Therefore the data transmission capacity of the systems deteriorated. Hence, for keeping the distance data rate product constant one should reduce either data rate or fiber span. Therefore, the analysis of systems performance by bit error rate \& constellation suggests that the overall system performance with 512 sub-carriers operating at $20 \mathrm{Gbps}$ is fairly good and having tolerable bit error rate for the initial $150 \mathrm{Km}$ and afterward it degrades.
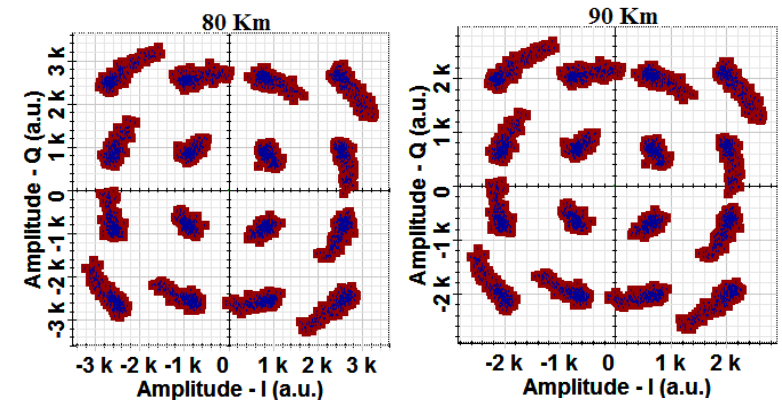

Figure4.10:Constellation for the Received Data Signal for OFDM 16-QAM 256 Sub-carriers at 20 Gbps.

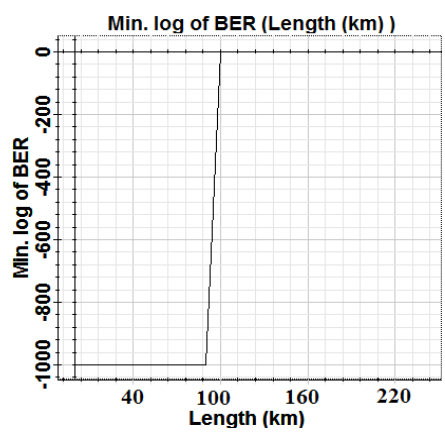

Figure4.11:Evaluation of BER V/s Length Obtained using OFDM 16-QAM 512 Sub-carriers at 20 Gbps.

\subsubsection{QAM OFDM 1024 Sub-carriers 20GBPS}

The various results are computed and presented graphically in the Figures 4.12-4.13 as constellation diagrams / BER graphs for the system at different fiber lengths from 0-200 Km at the centre wavelength of $1550 \mathrm{~nm}$, with using OFDM. From the constellation diagrams for the optical systems with 1024 sub-carriers transmission with fiber span ranging 0-200 $\mathrm{Km}$ for data rate $20 \mathrm{Gbps}$, it is found that for initial $100 \mathrm{Km}$ quality of the received signal is reasonably good and afterward it gets distorted due to the dispersion. The various symbols in constellation gets closer \& closer, causing the higher bit error rate, which in turn it limits the transmission performance of the system. Simultaneously, the BER also deteriorates as shown if the Figure 4.13. Subsequently this distortion due to the dispersion causes higher bit error rate. Therefore the data transmission capacity of the systems deteriorated. Hence, for keeping the distance data rate product constant one should reduce either data rate or fiber span. Hence from the system performance evaluation by BER and Constellation, it can be observe that the overall system performance at $20 \mathrm{Gbps}$ with using 1024 sub-carriers is well within the acceptable level with permissible bit error rate even for $100 \mathrm{Km}$ of the fiber span for the data rate of $20 \mathrm{Gbps}$ system with using OFDM, beyond $110 \mathrm{Km}$ the performance is degraded.
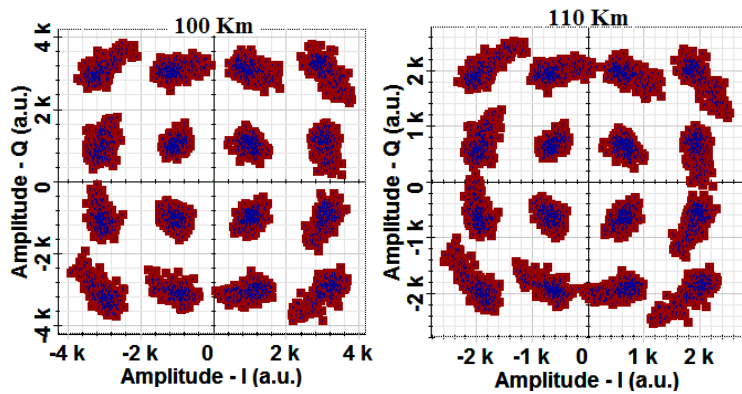

Figure4.12:Constellation for the Received Data Signal for OFDM 16-QAM 1024 Sub-carriers at 20 Gbps.

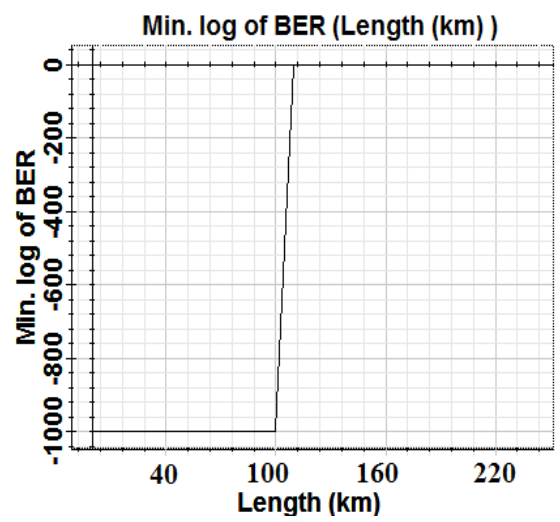

Figure4.13: Evaluation of BERV/s Length Obtained Through OFDM 16-QAM 1024 Sub-carriers at 20 Gbps.

\subsubsection{QAM OFDM 256 Sub-carriers 30GBPS}

From The various results are computed and presented graphically in the Figures 4.14-4.15 as constellation diagrams / BER graphs for the system at different fiber lengths from 0$200 \mathrm{Km}$ at the centre wavelength of $1550 \mathrm{~nm}$, without using OFDM. From the constellation diagrams for $30 \mathrm{Gbps}$ data rate 
with 256 sub-carriers transmission with selected fiber length of $0-200 \mathrm{Km}$, it analyzed that up to $80 \mathrm{Km}$ the signal quality is reasonably good and as we further increase the length of the fiber the received signal gets distorted due to the dispersion. The various symbols in the constellation diagram becomes closer \& closer, results in the higher bit error rate, which in turn it limits the transmission performance of the system. Simultaneously, the BER also deteriorates as shown if the Figure 4.15. Subsequently this distortion due to the dispersion causes higher bit error rate. Therefore the data transmission capacity of the systems deteriorated. Hence, for keeping the distance data rate product constant one should reduce either data rate or fiber span. Therefore, the analysis of systems performance by bit error rate \& constellation suggests that the overall system performance with 256 sub-carriers operating at $30 \mathrm{Gbps}$ is fairly good and having tolerable bit error rate for the initial $150 \mathrm{Km}$ and afterward it degrades.

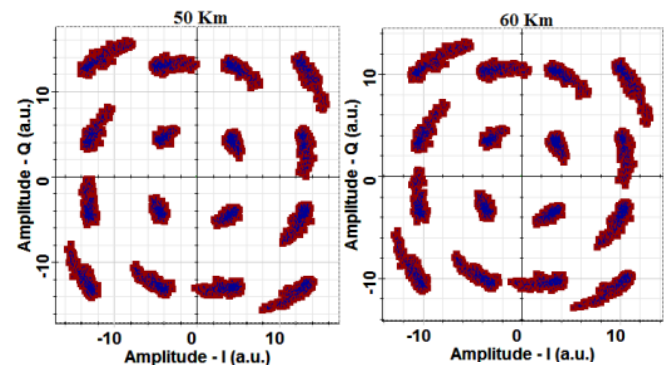

Figure4.14:Constellation for the Received Data Signal for OFDM 16-QAM 256 Sub-carriers at 30 Gbps.

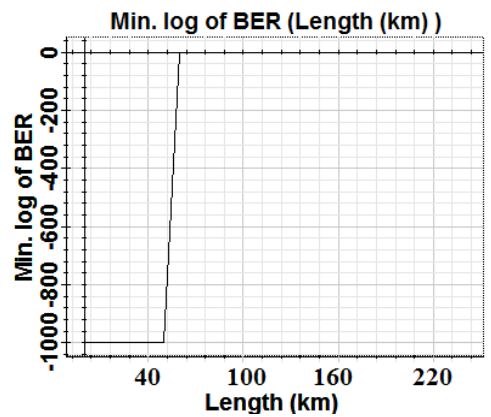

Figure4.15: Evaluation of BER V/s Length Obtained Through OFDM 16-QAM 256 Sub-carriers at 30 Gbps.

\subsubsection{QAM OFDM 512 Sub-carriers 30GBPS}

The different results are computed and shown graphically in the Figures 4.16-4.17 as constellation diagrams / BER graphs for the system at different fiber lengths from 0-200 Km at the centre wavelength of $1550 \mathrm{~nm}$, with / without using OFDM. From the constellation diagrams for the optical systems with 512 sub-carriers transmission with fiber span ranging $0-200 \mathrm{Km}$ for data rate $30 \mathrm{Gbps}$, it is found that for initial $70 \mathrm{Km}$ quality of the received signal is reasonably good and afterward it gets distorted due to the dispersion. The various symbols in constellation gets closer \& closer, causing the higher bit error rate, which in turn it limits the transmission performance of the system. Simultaneously, the BER also deteriorates as shown if the Figure 4.17. Subsequently this distortion due to the dispersion causes higher bit error rate. Therefore the data transmission capacity of the systems deteriorated.
Hence, for keeping the distance data rate product constant one should reduce either data rate or fiber span. Hence from the system performance evaluation by BER and Constellation, it can observe that the overall system performance at $30 \mathrm{Gbps}$ with using 512 sub-carriers is well within the acceptable level with permissible bit error rate even for $70 \mathrm{Km}$ of the fiber span and afterward it degrades.
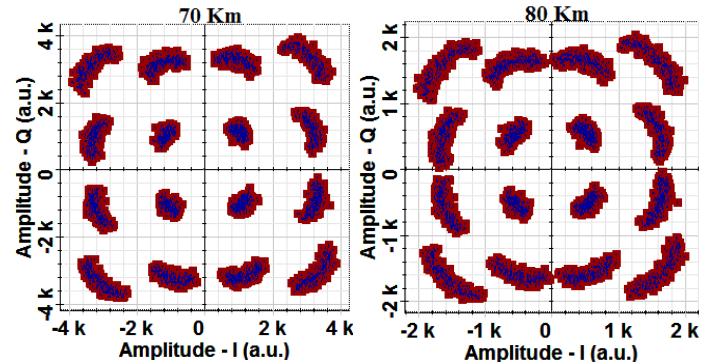

Figure4.16:Constellation for the Received Data Signal for OFDM 16-QAM 512 Sub-carriers at 30 Gbps.

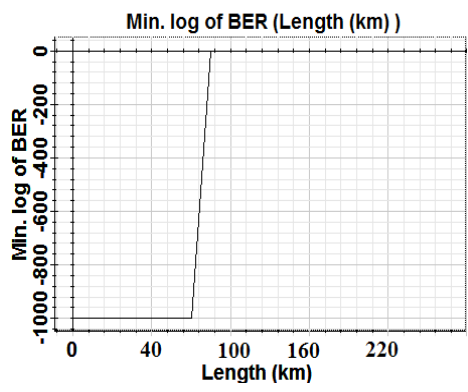

Figure4.17: Evaluation of BER V/s Length Obtained Through OFDM 16-QAM 512 Sub-carriers at 30 Gbps.

\subsubsection{QAM OFDM 1024 Sub-carriers 30GBPS}

The various results are computed and presented graphically in the Figures 4.18-4.19 as constellation diagrams / BER graphs for the system at $30 \mathrm{Gbps}$ for fiber span ranging from $0-200 \mathrm{Km}$ at $1550 \mathrm{~nm}$, with using OFDM sub-system. From the constellation diagrams for the optical systems with 1024 sub-carriers transmission with fiber span ranging 0-200 Km for data rate $30 \mathrm{Gbps}$, it is found that for initial $70 \mathrm{Km}$ quality of the received signal is reasonably good and afterward it gets distorted due to the dispersion. The various symbols in constellation gets closer \& closer, causing the higher bit error rate, which in turn it limits the transmission performance of the system. Simultaneously, the BER also deteriorates as shown if the Figure 4.19. Subsequently this distortion due to the dispersion causes higher bit error rate. Therefore the data transmission capacity of the systems deteriorated.

Hence, for keeping the distance data rate product constant one should reduce either data rate or fiber span. Hence from the system performance evaluation by BER and Constellation, it can observe that the overall system performance at $30 \mathrm{Gbps}$ with using 1024 sub-carriers is well within the acceptable level with permissible bit error rate even for $70 \mathrm{Km}$ of the fiber span for the data rate of $30 \mathrm{Gbps}$ system with using OFDM, beyond $80 \mathrm{Km}$ the performance is degraded. 

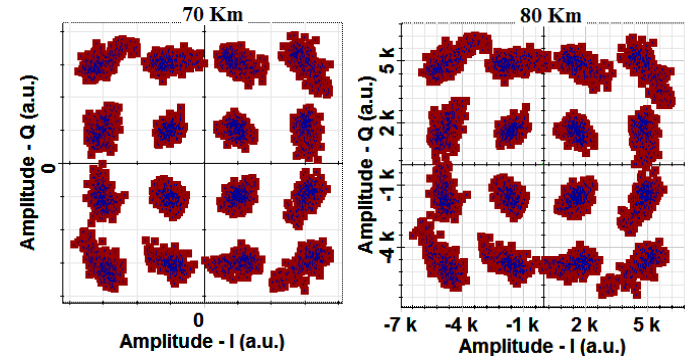

Figure4.18:Constellation for the Received Data Signal for OFDM 16-QAM 1024 Sub-carriers at 30 Gbps.

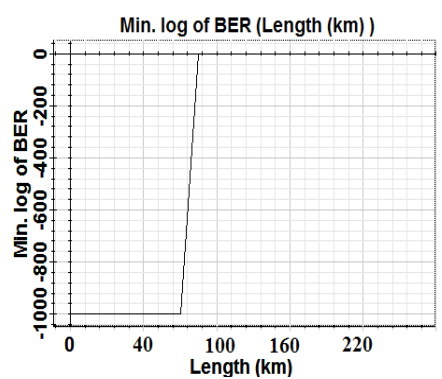

Figure4.19: Evaluation of BER V/s Length Obtained Through OFDM 16-QAM 1024 Sub-carriers at 30 Gbps.

\subsubsection{QAM OFDM 256 Sub-carriers 40GBPS}

The various results computed and presented graphically in the Figures 4.20-4.21 as constellation diagrams / BER graphs for the system at different fiber lengths from $0-200 \mathrm{Km}$ at the centre wavelength of $1550 \mathrm{~nm}$, without using OFDM. From the constellation diagrams for $40 \mathrm{Gbps}$ data rate with 256 subcarriers transmission with selected fiber length of 0-200 Km, it analyzed that up to $20 \mathrm{Km}$ the signal quality is reasonably good and as we further increase the length of the fiber the received signal gets distorted due to the dispersion. The various symbols in the constellation diagram becomes closer $\&$ closer, results in the higher bit error rate, which in turn it limits the transmission performance of the system. Simultaneously, the BER also deteriorates as shown if the Figure 4.21. Subsequently this distortion due to the dispersion causes higher bit error rate. Therefore the data transmission capacity of the systems deteriorated.

Hence, for keeping the distance data rate product constant one should reduce either data rate or fiber span. Hence from the system performance evaluation by BER and Constellation, it can observe that the overall system performance at $40 \mathrm{Gbps}$ with using 256 sub-carriers is well within the acceptable level with permissible bit error rate even for $30 \mathrm{Km}$ of the fiber span for the data rate of 40 Gbps system with using OFDM, beyond $30 \mathrm{Km}$ the performance is degraded.

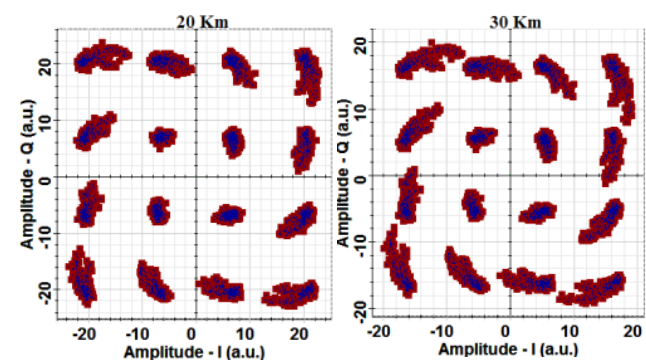

Figure4.20: Constellation for the Received Data Signal for OFDM 16-QAM 256 Sub-carriers at 40 Gbps.

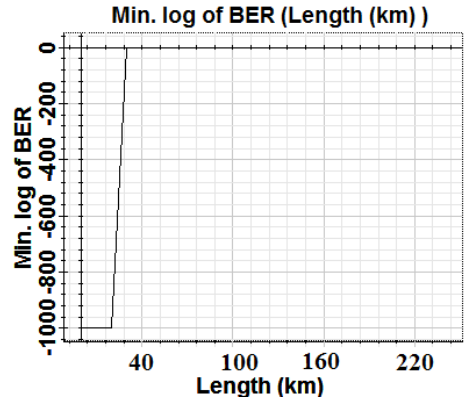

Figure4.21: Evaluation of BER V/s Length Obtained Through OFDM 16-QAM 256 Sub-carriers at 40 Gbps.

\subsubsection{6-QAM OFDM 512 Sub-carriers 40GBPS}

The different results are computed and shown graphically in the Figures 4.22-4.23 as constellation diagrams / BER graphs for the system at different fiber lengths from 0-200 Km at the centre wavelength of $1550 \mathrm{~nm}$, with / without using OFDM. From the constellation diagrams for the optical systems with 512 sub-carriers transmission with fiber span ranging $0-200 \mathrm{Km}$ for data rate $40 \mathrm{Gbps}$, it is found that for initial $20 \mathrm{Km}$ quality of the received signal is reasonably good and afterward it gets distorted due to the dispersion. The various symbols in constellation gets closer \& closer, causing the higher bit error rate, which in turn it limits the transmission performance of the system. Simultaneously, the BER also deteriorates as shown if the Figure 4.23. Subsequently this distortion due to the dispersion causes higher bit error rate. Therefore the data transmission capacity of the systems deteriorated. Hence, for keeping the distance data rate product constant one should reduce either data rate or fiber span.

Hence from the system performance evaluation by BER and Constellation, it can observe that the overall system performance at $20 \mathrm{Gbps}$ with using 512 sub-carriers is well within the acceptable level with permissible bit error rate even for $20 \mathrm{Km}$ of the fiber span for the data rate of $40 \mathrm{Gbps}$ system with using OFDM, beyond $30 \mathrm{Km}$ the performance is degraded.
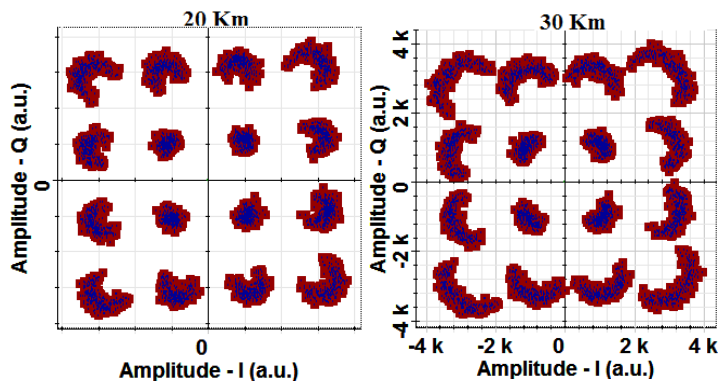

Figure4.22:Constellation for the Received Data Signal for OFDM 16-QAM 512 Sub-carriers at 40 Gbps. 


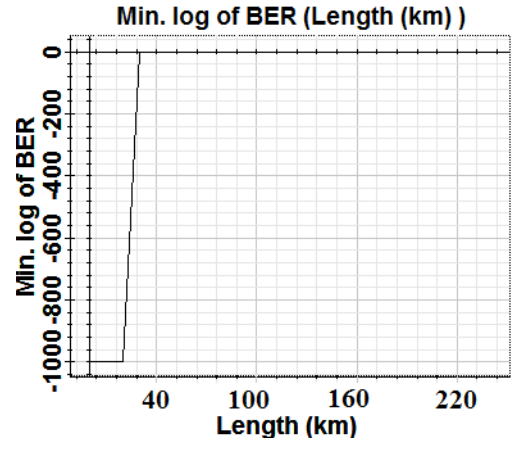

Figure4.23: Evaluation of BER V/s Length Obtained Through OFDM 16-QAM 512 Sub-carriers at 40.

\subsubsection{6-QAM OFDM 1024 Sub-carriers 40GBPS}

The various results are computed and presented graphically in the Figures 4.24-4.25 as constellation diagrams / BER graphs for the system at different fiber lengths from $0-200 \mathrm{Km}$ at the centre wavelength of $1550 \mathrm{~nm}$, without using OFDM. From the constellation diagrams for $40 \mathrm{Gbps}$ data rate with 1024 sub-carriers transmission with selected fiber length of 0-200 $\mathrm{Km}$, it analyzed that up to $20 \mathrm{Km}$ the signal quality is reasonably good and as we further increase the length of the fiber the received signal gets distorted due to the dispersion. The various symbols in the constellation diagram becomes closer \& closer, results in the higher bit error rate, which in turn it limits the transmission performance of the system. Simultaneously, the BER also deteriorates as shown if the Figure 4.25. Subsequently this distortion due to the dispersion causes higher bit error rate. Therefore the data transmission capacity of the systems deteriorated and for keeping the distance data rate product constant one should reduce either data rate or fiber span.

Hence from the system performance evaluation by BER and Constellation, it can observe that the overall system performance with using 1024 sub-carriers at $40 \mathrm{Gbps}$ is reasonably good with acceptable bit error rate for initial 20 $\mathrm{Km}$ andbeyond $30 \mathrm{Km}$ it is deteriorate.
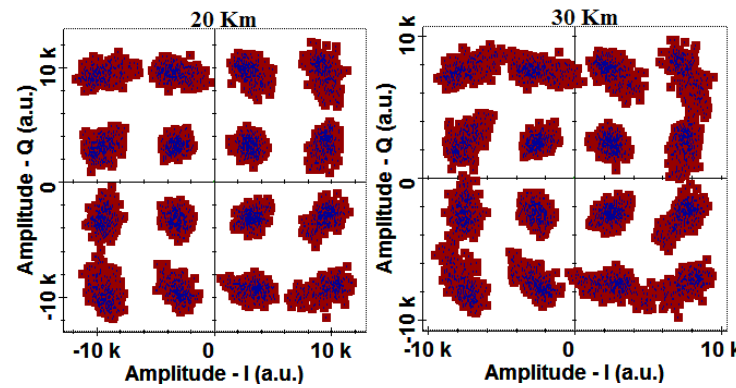

Figure4.24:Constellation for the Received Data Signal for OFDM 16-QAM 1024 Sub-carriers at 40 Gbps.

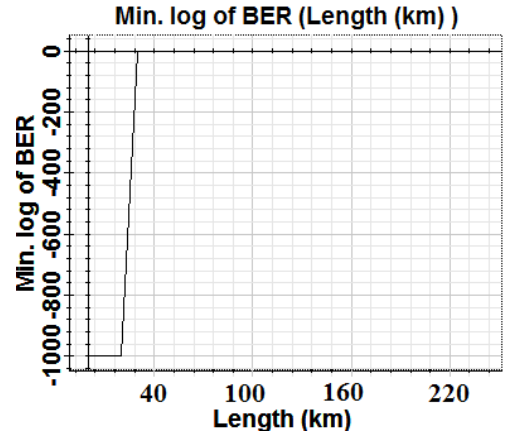

Figure4.25:Evaluation of BER V/s Length Obtained Through OFDM 16-QAM 1024 Sub-carriers at 40 Gbps.

\subsection{Performance evaluation of Optical Communication Systems by employing 16- QAM without using OFDM}

The performance of optical communication system without OFDM is evaluated here for comparisons purpose with fiber span ranging $0-50 \mathrm{Km}$, data transmission rate varying 1-10 Gbps. The results are depicted graphically as bit error rate and constellations. The transmitted 16-QAM constellations are shown in Figure 5.1. The 16-QAM encoded PRBS data signal propagated through optical fiber channel and received by at the receiving end by optical receiver. For the system performance evaluation,various parameters of the received signal are evaluated.

The system transmission performance is assessed in terms of constellation and bit error rate plot for varying fiber span, data rate, and different parameters. The various constellations diagram explicitly depicts that as the fiber span rises,different data symbols in constellation gets merged and deteriorates the systems data transmission capacity due to dispersion. Hence for mitigating dispersion effect andmaintaining constant data rate distance product one must reduce either data rate or fiber span.

\subsubsection{6-QAM 1.0 Gbps}

The various performance results are evaluated and presented graphically in the Figures4.26-4.27 as constellation diagrams and bit error rate plots fiber span varying 0-100 Km at centred1550 nm wavelength, without using OFDM.From the constellation diagrams at 1 Gbps with 16-QAM modulation, it is observed that for initial $40 \mathrm{Km}$ the signal quality is reasonably good and afterward it gets distorted due to the dispersion. The different symbols in the constellation diagram gets closer \& closer, causing increase in bit error rate, subsequently limits the system transmission performance. At the same time, the BER also degrades as shown in the Figure 4.27. Subsequently this distortion due to the dispersion causes higher bit error rate. Therefore the data transmission capacity of the systems deteriorated. Hence, for maintaining constant distance data rate product one must reduce either data rate or fiber span.

Therefore, from the system performance assessmentby bit error rate and constellation it can adduce that the overall performance of the system with 16-QAM modulation is well within the acceptable level with permissible bit error rate even for $40 \mathrm{Km}$ of the fiber length for the data rate of $1.0 \mathrm{Gbps}$ after50 $\mathrm{Km}$ it is degraded.From different results it can be deduce that raise in the fiberlength and data rate results in distorted received signal. The different results also depict that for the system without OFDM product of the distance data 
rate comparatively smaller. It can be observe that the permissible bit error rate can be achieved even for $40 \mathrm{Km}$ of the fiber length for the system. It can be conclude that the systems using OFDM give better results compare to without OFDM.

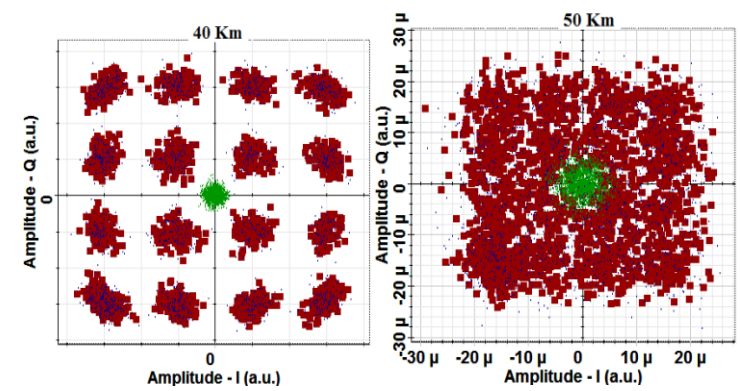

Figure4.26: Constellation for the Received Data Signalfor 16-QAM without OFDM at 1.0 Gbps.

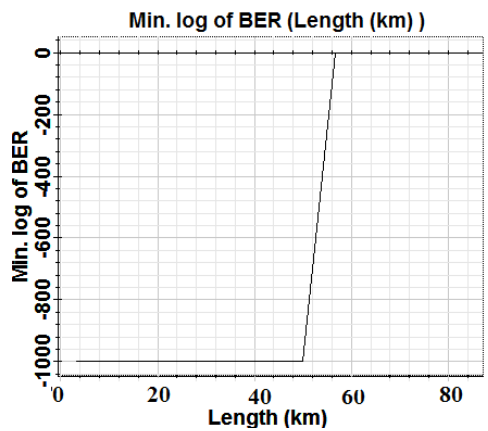

Figure4.27: Evaluation of BER V/s Length for 16-QAM without OFDM at 1.0 Gbps.

\subsubsection{6-QAM 5.0 Gbps}

The various results are computed and presented graphically in the Figures4.28-4.29 as constellations/BER plot for the optical system without using OFDM for various fiber lengths from 0$100 \mathrm{Km}$ at wavelength of operation centred at $1550 \mathrm{~nm}$.From the depicted constellations, it analysedthat up to $30 \mathrm{Km}$ the received signal quality is satisfactorily and afterwards the signal quality deteriorates because of the dispersion. The constellations shows that the various symbol get merged, resulting in the higher bit error rate, which in turn it limits the transmission performance of the system. Simultaneously, the BER also deteriorates as shown in the Figure 4.29. Subsequently inter-symbol interference due to dispersion increases bit error rate.

Hence, the data transmission capacity of the systems deteriorated. Therefore, for keeping the distance data rate product constant one should reduce either data rate or fiber span. Hence from the examination of the system performance by Constellations and BERit seems that the system performance is acceptable and within permissible bit error rate even for $30 \mathrm{Km}$ of the fiber length, afterwardit is deteriorates.Various resultsindicate that distortion proportionately increases with the transmission distances and data rates.

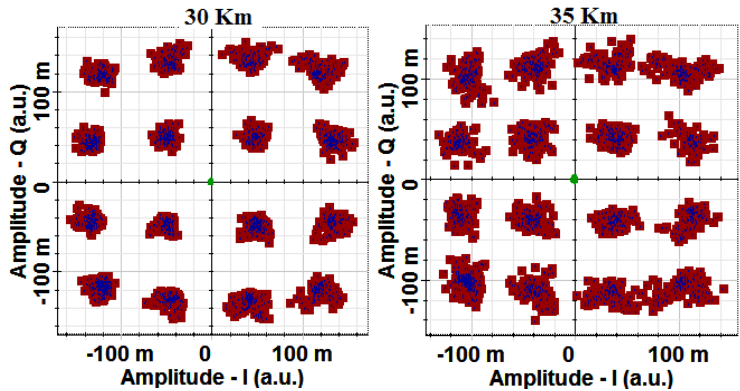

Figure4.28:Constellation for the Received Data Signal for 16-QAM without OFDM at 5.0 Gbps.

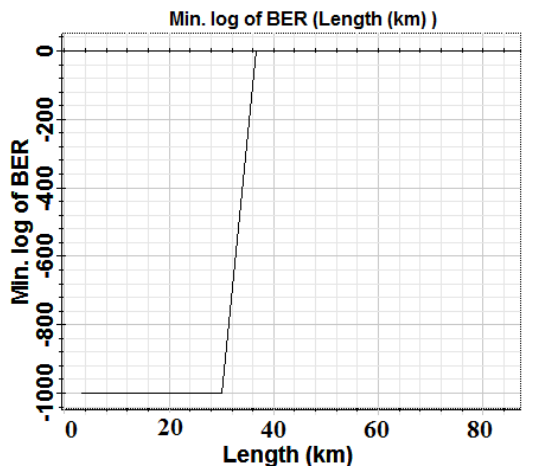

Figure4.29: Evaluation of BER V/s Length for 16-QAM without using OFDM at 5.0 Gbps.

\subsubsection{6-QAM 10 Gbps}

The various performance results are evaluated and presented graphical plotsin Figures4.30-4.31 as constellation diagrams and bit error rate for fiber span varying $0-100 \mathrm{Km}$ at centred $1550 \mathrm{~nm}$ wavelength, without using OFDM , at $10 \mathrm{Gbps}$ with 16-QAM modulation. From the results, it can be adduce that for initial $15 \mathrm{Km}$ the received signal quality is fairly good and afterward it gets distorted due to the dispersion. The different symbols in the constellation diagram gets closer \& closer, causing increase in bit error rate, subsequently limits the system transmission performance. At the same time, the BER also degrades as shown in the Figure 4.31. Subsequently this distortion due to the dispersion causes higher bit error rate. Therefore the data transmission capacity of the systems deteriorated.

Hence, for maintaining constant distance data rate product one must reduce either data rate or fiber span. Therefore, from the examination of the system transmission performance by BER and constellation, it is observe that the overall system performance with 16-QAM modulation is well within the acceptable level with permissible bit error rate even for fiber span of $15 \mathrm{Km}$ for the data rate of $1.0 \mathrm{Gbps}$ without using OFDM, after $20 \mathrm{Km}$ the performance is degraded. From the different results one can adduce that raise in the fiber length \& data rate causes in corrupted received signal. Also it shows that for the system without OFDM product of the distance data rate comparatively smaller and permissible bit error rate can be achieved even for $15 \mathrm{Km}$ of the fiber length. It can be deduce that by using OFDM, systems give better results compare to without using OFDM. 

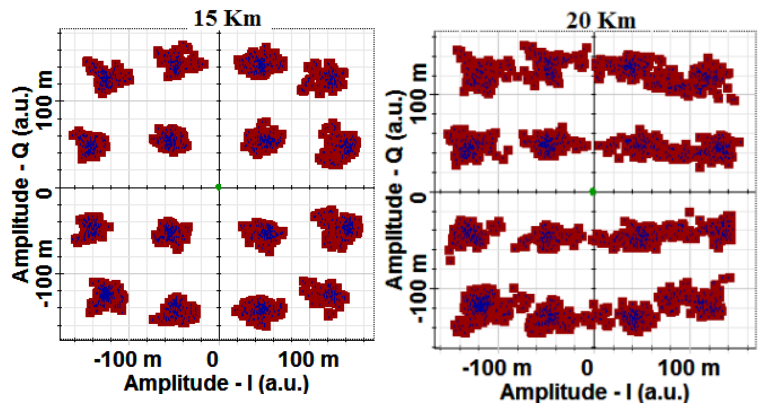

Figure4.30: Constellation for the Received Data Signal for 16-QAM at 10 Gbps without OFDM.

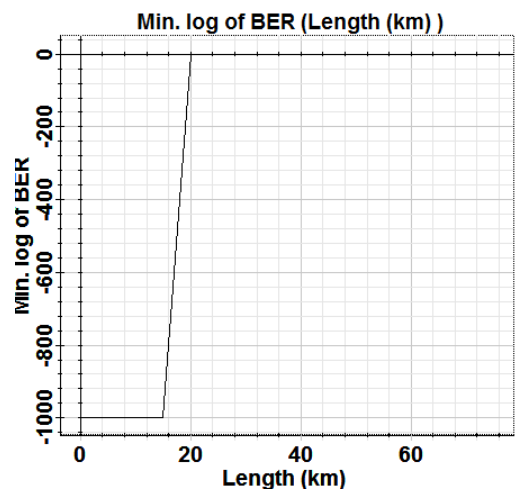

Figure4.31: Evaluation of BER V/s Length for 16-QAM at without OFDMsystem at 10 Gbps.

\section{CONCLUSIONS AND FUTURE WORK}

For evaluating the impact of the OFDM to mitigate the dispersion a comparative performance investigation of the dispersion compensated optical fiber communication system with using OFDM conducted. Optical fiber systems have been configured for the evaluation of the performance with and without using OFDM. Various simulated resultsdepicts that the systems transmission performance are entirely dependent upon a proper selection of data rate, transmission distance, and modulation schemes etc. The systems data rate ranging 1.0-40 Gbps, at the centre wavelength $1550 \mathrm{~nm}$ and fiber span ranging $0-200 \mathrm{Km}$, the performance have been evaluated by computing the signal constellations / BER and compared at the receiver for different fiber lengths, data rate, and number of sub-carriers etc. From the different simulated results it can be seen that increase in the fiber length and data transmission rate results in signal distortion. At the same time, the BER also deteriorates. Therefore the data transmission capacity of the systems deteriorated.

Hence, for maintaining the constant distance data rate product one should reduce either data rate or fiber span. It can be adduce that the systems with using OFDM give better results compare to system without using OFDM. Also, different results represent that for the system without OFDM distance data rate product is comparatively smaller. It can be observe that the permissible bit error rate can be achieved even for fiber length of $160 \mathrm{Km}$ for the system at $10 \mathrm{Gbps}$ with using OFDM.Also, different results of this work, indicates that the OFDM is superior dispersion compensation technique for mitigating the dispersion in system with higher spectral efficiency. OFDM can favorably compensate the dispersion in the system without using any separate dispersion compensating sub systems. Additionally, the performance of the optical communication system using OFDM can be elevated by integrating various error detecting and correcting techniques in the system and optimizing OFDM parameters. The main drawbacks for OFDM systems are high value of peak to average power ration and the susceptibility for frequency offset and phase noise. For taking complete benefit and mitigate the negative consequences of the OFDM a careful blueprint utilizing various aspects of the OFDM including the susceptibility to phase noise and frequency offset, the peak to average power ratio, distortion due to clipping of peak power, synchronization error, error due frequency instability etc. can be studied later on as part of future work, since this work has become absolutely extensive.

From the various simulated outcome it can be inferred that the performance of optical communication system with OFDM is far better than without OFDM, under realistic channel condition. The transmission distance was found to be less affected by dispersion in the fiber up to bit rate of few Gbps. A dramatic decrease in the transmission distance is observed when the bit rate increases further and the system becomes dispersion limited. Furthermore, by employing large number of sub-carrier, using advance sub-carriers modulation schemes andaddition of cyclic prefix can give excellent transmission performance for long distance high data rate transmission.From the examination of the constellation diagrams for data rate 10-40 Gbps with fiber span ranging 0$200 \mathrm{Km}$; it is observed that as fiber length increases signal getsdistortion due to the impact of the dispersion. The symbols in the constellation get merged and causing higher bit error rate, therebylimits the data transmission performance.For low data rate transmission the systems has shown better performance through constellation pattern but as the data rate increases it has shown degrading effect. Bit error rate have been calculated and observed at different values of transmitted distance, at the receiving end and results for bit error rate v/s length shows that bit error rate, which is a significant transmission performance parameter degrades with increase in transmission length.

From the performance evaluation for the optical system with using OFDM by employing 16-QPSK 512 sub-carriers systems at $10 \mathrm{Gbps}$, it can be observe that the BER deteriorates severely after $160 \mathrm{Km}$ the dispersion degrades the performance. Hence, the proposed system is suitable for 160 $\mathrm{Km}$ of transmitted distance. Therefore, it can adduce that the tolerable bit error rate can be obtained even for $160 \mathrm{Km}$ of transmission distance with data rate of $10 \mathrm{Gbps}$ for systems with OFDM.Hence it can be concluded that as the distance progressive BER deteriorates. Improvement in BER rate can be achieved by increasing number of OFDM carriers, cyclic prefix length and incorporating FEC technique in the system. Consequently it can be concluded that transmission performance of OFDM system is dependent upon number of transmission parameters like data rate, number of sub-carriers and modulation schemes used. The effects of using varying number of sub-carriers have also been investigated from the various results we observe that an increase in the number of sub-carrier from 256 to 1024 results in the improvement in the performance. The use of a large number of sub-carriers has been observed to be more effective in overcoming the fibre dispersion.According to the simulations results, OFDM seems to be better modulation scheme for mitigating the impact of dispersion with efficiently utilizing bandwidth, which does not needed external components for the dispersion compensation. It can compensate for almost any practically accumulated dispersion without introducing a dispersion 
penalty, which introduces nonlinearity. Therefore, it is an adaptive method which is especially suitable for optical networks with dynamic transmission path.This demonstrates that the optical systems with using OFDM are the strongest methods to eliminate the impact of the dispersion causing inter-symbol interference.OFDM can effectively mitigate the impact of dispersion without employing any separate dispersion compensating module or sub-system. Here, different simulated results suggest that at $10 \mathrm{Gbps}$ for a distance up to $160 \mathrm{KM}$, it gives reasonably good quality of transmission without using any external compensation module.The results also reveal that systems with using OFDM havecomparatively better performance in terms of extended transmission distance and needed data rate. It is in agreement with the distance data rate product that can be obtained which is appropriate for next generation networks. Use of advance modulation schemes for sub-carriers, increase in number of sub-carriers, insertion of guard band along with the forward error correction coding will steer to extra higher data rate.

\section{REFERENCES}

[1] Gruner Nielsen L., at al., "Dispersion Compensating Fibers," Journal of Lightwave Technology, vol. 23, no. 11, pp.3566-3579, Nov 2005.

[2] Kenichi Kitayama et al., "Dispersion Effects of FBG Filter and Optical SSB Filtering in DWDM Millimeter Wave Fiber Radio System," Journal of Lightwave Technology, vol. 20, no.8, pp. 1397-1407 Aug 2002.

[3] Sethi R.K. andGoel Aditya, "Performance Analysis of Optical Communication System using OFDM by employing QPSK Modulation," IJRITCC: International Journal on Recent and Innovation Trends in Computing and Communication, vol. 3, no. 1, pp.226-237 Jan 2015.

[4] Michael S. Borella et al., "Optical Components for WDM Light wave Networks," Proceedings of IEEE, vol. 85, pp. 1274-1305, 1997

[5] Van De Beek J.J., Sandell M. and Borjesson P. O., "ML Estimation of Time and Frequency Offset in OFDM System," IEEE Trans. Signal Process, vol.45, no. 12, pp. 1800-1805, Jul. 1997.

[6] Birks T.A., Mogilevtsev D. and Russell P. St. J., "Group Velocity Dispersion in Photonic Crystal Fibers," Optics Lett., vol. 23, pp. 1662-1664, 1998

[7] Lu H., "Performance Comparison between DCF and RDF Dispersion Compensation in Fiber Optical CATV System," IEEE Trans. Broadcast. vol. 48,no. 4, pp. 370-373, 2002.

[8] Bryn J. Dixon, Roger D. Pollard and Stavros Iezekiel, "Orthogonal Frequency Division Multiplexing in wireless Communication System with Multimode Fiber Feeds," IEEE Transactions on Microwave Theory and Techniques, vol. 49, no. 8, Aug 2001.

[9] Sethi R.K. and Goel Aditya, "Integrated Optical Wireless Network for Next Generation Wireless System," Signal Processing: an International Journal (SPIJ),vol. 3, no. 1, pp. 1-13, Jan/Feb 2009.

[10] Goel Aditya, et al., "Performance Analysis of Continuous Wavelength Optical Burst Switching Networks," International Journal of Engineering (IJE), vol. 3, no.6, pp. 609-621, Nov/Dec 2009.

[11] Sethi R.K. and Goel Aditya, "Performance Analysis of High Capacity Integrated Fiber Radio Communication
System,"Proc. of SPIE: Broadband Access Communication Technologies,vol. 6390 pp. 63900J-1 to $63900 \mathrm{~J}-11$, Oct 2006.

[12] Kao K.C. and Hockman G.A., "Dielectric Fiber Surface Waveguides for Optical Frequencies," Proc. IEE, vol. 133, pp. 1151-1 158, July 1966.

[13] Kapron F.P., Keck D.B. and Maurer R.D., "Radiation Losses in Glass Optical Waveguides," Appl. Phys. Lett., vol. 17, pp. 423-425, Nov. 1980.

[14] Lin C., "Optical Fiber Transmission TechnologyHandbook of Microwave and Optical Components,' $2^{\text {nd }}$ Ed., John Wiley, 1991.

[15] Nielsen T.N., et al., "Dynamic Post Dispersion Optimization at $40 \mathrm{~Gb} / \mathrm{S}$ using a Tunable Fiber Bragg Grating," IEEE Photo. Tech. Lett., vol. 12, no. 2, pp. 173-175, Feb. 2000.

[16] Agrawal. G. P., "Fiber Optic Communications System," $2^{\text {nd }}$ Ed.,John Wiley \& Sons, New York.

[17] Gisin N. and Pellaux J., "Polarization Mode Dispersion: Time versus Frequency Domains," Optical Commun. vol. 89, no. 2-4, pp. 316-323, May 1992.

[18] Grüner Nielsen L., et al., "Dispersion Compensating Fiber," Optical Fiber Technology, vol. 6, no. 2, pp.164180, April 2000.

[19] Knudsen S.N. and Veng T., "Large Effective area Dispersion Compensating Fiber for Cabled Compensation of Standard Single Mode fiber," Proceeding of Optical Fiber Communication Conference (OFC) 2000, vol. 1, pp. 98-100, Paper Tug5, March 2000.

[20] Knudsen S.N., "Design and Manufacture of Dispersion Compensating Fiber and Their Performance In System," Proceeding of the Optical Fiber Communication Conference (OFC) 2002, Paper Wu3, pp. 330-332, March 2002

[21] Le Q.N.T., Veng T., and Grüner Nielsen L., "New Dispersion Compensating Module for Compensation of Dispersion and Dispersion Slope of Non-Zero Dispersion fibre in the C-Band," Proceeding of Optical Fiber Communication Conference (OFC) 2001, Paper Tuh5, March 2001.

[22] Williams J.A.R., et al., "Fibre Dispersion Compensation using a Chirped in fibre Bragg Grating," Electronics Letters, vol. 30, no. 12, pp. 985-987, June 1994.

[23] Lima M.J.N., Teixeira A.L.J., and Da Rocha J.R.F., "Simultaneous filtering and Dispersion Compensation in WDM System using Apodised fibre Gratings," Electronics Letters, vol. 36, no. 16, pp. 1412-1414, Aug 2000.

[24] Morin M., Poulin M., Mailloux A., Trépanier F., and Painchaud Y., "Full C-Band Slope Matched Dispersion Compensation Based on a Phase Sampled Bragg Grating," Proceeding of Optical Fiber Communication Conference, (OFC) 04, Paper Wk1, Feb 2004.

[25] Flanders D. C., Kogelnik H.,Schmidt R. V. and Shank C. V., "Grating Filters for Thin Film Optical Waveguides," Applied Physics Letters, vol. 24, pp. 194 196, 1974

[26] Measures R., Alavie T., Karr S. and Coroy T., "Smart Structure Interface Issues and their Resolution: Bragg 
Grating Laser Sensor and the Optical Synapse," Proc. SPIE, vol. 1918, 1993.

[27] Xiaodong Wang, "OFDM and its Application to 4G," Proceeding of the 14th Annual Wireless and Optical Communications Conference, USA, April, 2005.

[28] Mcghan D., Laperle C., Savchenko A., Chuandong Li, Mak G. and O'sullivan M., "5120 Km RZ-DPSK Transmission over G652 Fiber at $10 \mathrm{~Gb} / \mathrm{S}$ with no Optical Dispersion Compensation," Proceeding of the Conference on Optical Fiber Communication, vol. 6, pp. 79-81, 2005.

[29] Zou W.Y. and Wu Y., "COFDM: an Overview," IEEE Trans. Broadcast., vol.41, pp. 1-8, Mar. 1995.

[30] https://www.optiwave.com

[31] R. Chang, "Orthogonal Frequency Multiplex Data Transmission System," $2^{\text {nd }}$ Ed. John Wiley \& Sons, 605 Third Avenue, New York.

[32] Sari H., Levy Y. and Karam G., "An Analysis of Orthogonal Frequency Division Multiple Access," Proceeding of IEEE Globecom, pp. 1635-1649, Nov1997.

[33] Bingham J.A.C., "Multicarrier Modulation for Data Transmission: an Idea Whose Time has Come," IEEE Comm. Magazine, vol. 28, no. 5, pp. 5-14, 1990.

[34] Chow J., Tu J.and Cioffi J., "A Discrete Multi-tone Transceiver System for HDSL Applications," IEEE Journal on Selected Areas in Communications, vol. 9, no. 6, pp. 895-908, 1991.
[35] Weinstein S., "The History of Orthogonal Frequency Division Multiplexing," IEEE Communications Magazine, vol. 47, no. 11, pp. 26-35, 2009.

[36] Pan Q.and Green R., "Bit Error Rate Performance of Lightwave Hybrid AM/OFDM System with Comparison with AM/QAM System in the Presence of Clipping Impulse Noise," IEEE Photonics Technology Letters, Vol. 8, No. 2, pp. 278-280, 1996.

[37] Filios A.,et al. "16 Channel, 10 Gb/s DWDM Transmission of Directly Modulated Lasers with $100 \mathrm{GHz}$ Channel Spacing over $100 \mathrm{~km}$ of Negative Dispersion Fiber," Proc. LEOS 14th Annual Meeting, Paper ThK3, vol. 2, pp.742-743, Nov 2001.

[38] Yu and Chang G. K., "Generation and transmission of eight channel DWDM signals with 10 Gbitps payloads and 2.5 Gbitps labels over 200km SMF-28," IEEE Electronics Letters,vol.40, no.2, pp. 135-137, 2004.

[39] https://www.mathworks.com

[40] Sethi R.K. and Goel Aditya, "Dispersion Compensation in Optical Communication Systems by employing 16QAM Modulation using OFDM," IMPACT: International Journal of Research in Engineering \& Technology, vol. 3, no. 2, pp. 47-64, Feb 2015.

[41] DjordjevicI. .B. and VasicB., "Orthogonal Frequency Division Multiplexing for High-Speed Optical Transmission," Opt. Exp. vol. 14, no. 9, pp. 3767-3775, 2006. 